Classification

Physics Abstracts

$51.60-51.20$

\title{
Direct detection of spin waves in gaseous ${ }^{3} \mathrm{He} \uparrow$
}

\author{
G. Tastevin, P. J. Nacher, M. Leduc and F. Laloë \\ Laboratoire de Spectroscopie Hertzienne de l'E.N.S. $\left({ }^{*}\right)$, 24, rue Lhomond, 75005 Paris, France
}

(Reçu le 23 novembre 1984, accepté le 28 janvier 1985)

\begin{abstract}
Résumé. - Dans un gaz de ${ }^{3} \mathrm{He}$ polarisé, la diffusion de spin prend à basse température un caractère oscillant et engendre des ondes de spin; ces ondes, amorties, ont une surtension $\mu M$, où $M$ est le taux de polarisation nucléaire et $\mu$ un coefficient caractéristique de l'importance relative des effets d'échange lors des collisions binaires. Nous décrivons ici une technique RMN où ces modes oscillants sont directement excités et détectés par deux paires de bobines, chaque paire étant formée de deux enroulements en opposition afin de créer ou détecter un pur gradient d'aimantation dans l'échantillon. Les valeurs mesurées pour $\mu$ sont en bon accord avec la théorie et avec les expériences antérieures, mais seulement à des températures dépassant $2 \mathrm{~K}$; au-dessous, les mesures sont perturbées par des effets en partie incompris, et sans doute liés à la formation d'une monocouche de ${ }^{3} \mathrm{He}$ sur l'enduit d'hydrogène solide qui couvre la paroi de la cellule.
\end{abstract}

\begin{abstract}
In gaseous spin-polarized ${ }^{3} \mathrm{He}$ at low temperature, spin-diffusion becomes oscillatory and gives rise to spin waves; these waves have a quality factor $\mu M$, where $M$ is the (relative) nuclear magnetization and $\mu$ a dimensionless coefficient characteristic of the importance of exchange effects in binary collisions. We describe here an NMR technique where these oscillatory modes are directly excited and detected, with the use of two sets of radiofrequency coils (for induction and detection), each connected in opposition in order to create or detect pure magnetization gradients over the sample. The measurements of $\mu$ are in good agreement with theory as well as with previous experiments, but only at temperatures above $2 \mathrm{~K}$; below, the measurements are affected by processes which are not fully understood, probably related to the formation of a ${ }^{3} \mathrm{He}$ monolayer on the surface of the coating of solid hydrogen which covers the inner surface of the cell.
\end{abstract}

Spin diffusion and other transport phenomena may be substantially different in spin-polarized gases at low temperatures from ordinary, unpolarized, gases [1]. It is well known that, usually, spin diffusion is a purely dissipative process; it gives rise, in any particular geometry with appropriate boundary conditions, to a series of modes which are damped but do not oscillate (except, of course, for the trivial rotation of the spins due to their Larmor precession in the external field). This is not true for spin polarized gases such as ${ }^{3} \mathrm{He} \uparrow$ and $\mathrm{H} \uparrow$ at low temperatures, when exchange effects become important $[2,4]$ : each mode becomes oscillatory and can be described as a standing " spin wave " having a well-defined frequency and damping. In other words, the diffusion of spins in the gas, in addition to being a dissipative process, becomes also reactive; the ratio bet-

(*) Laboratoire associé au CNRS, no 18. 
ween the oscillation frequency and the damping rate of the modes (their " quality factor ") is simply the product $\mu M$ of a dimensionless coefficient $\mu$ by the relative magnetization of the gas $M\left({ }^{1}\right)$.

These spin oscillations are a pure quantum phenomenon due to particle indistinguishability. The reactive diffusion terms are introduced by the so called "identical spin rotation effect " occurring during binary collision between atoms. The same effect can also be seen, within the frame of Landau mean-field theory, as a consequence of the spin dependent part of the " molecular field $»[2,4,5]$; both points of view are indeed correct, but, as noted in reference [6], the advantage of the former is to include spin rotation effects in lateral collisions, which may play a non-negligible role. An exact calculation of $\mu$ for ${ }^{3} \mathrm{He} \uparrow$ and $\mathrm{H} \uparrow$ has been given by $\mathrm{C}$. Lhuillier [7].

Studying the properties of spin waves in a dilute gas can be compared to measuring a new transport property of the system, since $\mu$ is completely independent of the other transport coefficients. Recently, several experiments on spin waves in dilute, non-degenerate systems have been reported, in the case of $\mathrm{H} \uparrow[8,9]$, of very dilute solutions of ${ }^{3} \mathrm{He} \uparrow$ in superfluid ${ }^{4} \mathrm{He}$ (where the ${ }^{3} \mathrm{He}$ atoms behave very similarly to a real gas) [10] and ${ }^{3} \mathrm{He} \uparrow$ [11]. Those experiments used nuclear magnetic resonance in conditions where the signal detected is primarily sensitive to the average magnetization of the sample inside the NMR detection coils, while being rather insensitive to magnetization gradients. Now, in a uniform magnetic field, due to angular momentum conservation, the total magnetization of a gaseous sample cannot be affected by identical spin rotation (or molecular field) effects ; the spin waves actually correspond to spatially varying diffusion modes which have zero average value over the sample and cannot be directly detected by NMR. Consequently, in all those experiments, a gradient of the static magnetic field was necessary to couple the spin waves to the average magnetization of the sample; an " indirect " detection of their properties then became possible. Another equivalent point of view is to consider the spin diffusion modes as modified by the presence of a static magnetic gradient, and to note that they acquire a non-zero average value as soon as the gradient is present.

By contrast with this type of method, we present in this Letter a « direct " detection method where no static magnetic gradient is necessary. The idea is simply that, if NMR coils are connected in opposition, they create a pure RF field gradient with zero spatial average value, so that they directly excite (or detect) spin waves. Figure 1 gives a schematic view of the situation : two pairs of coils, each in opposition, are mounted around a spherical sample. The first pair is used for generating RF pulses and creates an initial space-dependent tilt of the nuclear magnetization, while the second picks up the induced electrical signal. In ordinary NMR, the axes of induction and pick-up coils are orthogonal to minimize their direct coupling; in our case, the " cross talk " is minimized when the axes are at $45^{\circ}$. Figure 1 shows why this happens, as well as the magnetization created just after a short pulse from the induction coils, which has a zero average value over the cell. It is clearly coupled to the pick-up coils provided that they are also in opposition.

This method has been used to detect spin waves and measure $\mu$ in gaseous spin-polarized ${ }^{3} \mathrm{He}\left({ }^{3} \mathrm{He} \uparrow\right)$. The experimental set-up is very similar to that described previously [11], except for the NMR coils which are set as described in the preceding paragraph. Optical pumping with a laser creates nuclear polarization in a gas at room temperature, while gas diffusion through a long glass pipe transfers the nuclear polarization to a second cell at liquid helium temperatures; in the lower part of the system, solid hydrogen is used as a cryogenic coating to suppress the strong nuclear relaxation which would be produced by bare glass walls at a few degrees Kelvin. Initial longitudinal nuclear polarizations of order $30 \%$ are obtained, and then partly transformed by a RF pulse into gradients of transverse magnetization. The experiment is done in conditions

( $\left.{ }^{1}\right)$ Exchange effects also introduce non-linearities in the equations of spin diffusion but, for the sake of simplicity, we assume here that the magnetization of the gas is almost uniform in space, so that a linearized theory of diffusion can be used. 


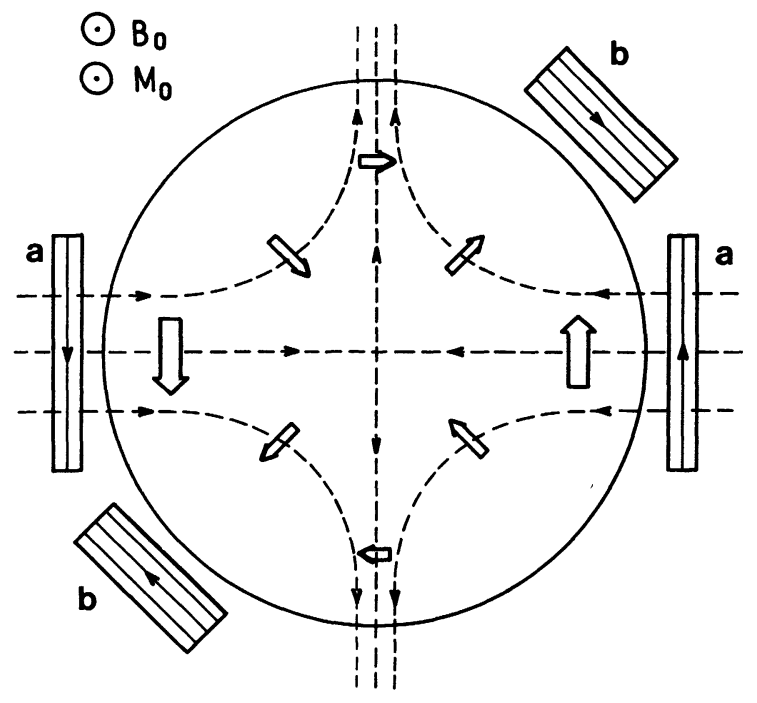

Fig. 1. - The induction coils (a) are connected in opposition and create an RF magnetic field with zero average value over the spherical sample, as shown by the magnetic field lines (dashed lines). The static field $\mathbf{B}_{0}$ and the initial magnetization are perpendicular to the plane of the figure. Just after a short resonant RF pulse, the direction of the transverse magnetization is purely inhomogeneous, as shown by the large open arrows in the figure and can be described by $l=1$ diffusion modes of the transverse magnetization. The detection coils (b), also in opposition, and with their axes at $45^{\circ}$ of the induction coils, receive a flux which is maximum at this initial time. For coils in opposition, the induction coupling in the absence of spins is minimized for a $45^{\circ}$ angle, instead of $90^{\circ}$ for coils in parallel.

where the transverse magnetization never exceeds a small fraction $(<10 \%)$ of the longitudinal one, so that a linearized spin diffusion theory $[3,8,9]$ can be used. After a short induction pulse, an exponentially decaying signal is received by the pick-up coils in opposition. If the balance of the detection coils is correct, the decay time constant of the signal is $\tau_{\mathrm{D}}$, the time constant associated with the lowest diffusion mode $n=l=1$ for perfectly reflecting boundary conditions $\left({ }^{2}\right)$; by contrast, it would be $T_{2}$, the transverse relaxation time, in ordinary NMR experiments where the quantity measured is the total transverse magnetization of the sample. In our experiments, the inner diameter of the cell is $1.8 \mathrm{~cm}$ and the number density of the gas $n \cong 2 \times 10^{18} \mathrm{~cm}^{-3}$, so that the value of $\tau_{\mathrm{D}}$ is $\tau \cong 200 \mathrm{~ms}\left({ }^{3}\right)$ (see Ref. [7] for a value of the spin diffusion coefficient $D$ ). On the other hand, the magnetic gradients are reduced with the help of superconducting shielding and sets of coils creating pure gradients of the longitudinal static field, as described in reference [11]; the motional narrowing conditions are fulfilled and in practice, values of $T_{2}$ ranging from $1 \mathrm{~s}$ to $10 \mathrm{~s}$, or more, are accessible. It is therefore not difficult to distinguish between the spin wave signal and a possible spurious signal due to the total transverse magnetization of the sample, which would decay with a much longer time constant.

${ }^{2}$ ) The diffusion modes corresponding to higher $n$ or $l$ values also contribute to the signal, but only weakly (see the Appendix of Ref. [21] for an evaluation of the coupling coefficients for creation and detection). Moreover, they are damped with shorter time constants.

$\left(^{3}\right)$ Since $|\mu M| \ll 1$ in our experimental conditions, we can neglect second order corrections and ignore the modifications of the diffusion times introduced by spin rotation effects (" blocking " of the transverse diffusion, which is second order in $\mu M$ ). 
The experimental procedure goes as follows : first, a spin wave signal is recorded during its decay, for about a second after a short inhomogeneous NMR pulse; then, the longitudinal magnetization is reversed with a $180^{\circ}$ pulse created by auxiliary induction coils (in parallel, as in usual NMR); a second spin wave signal is then recorded after a second inhomogeneous RF pulse, then the longitudinal magnetization is reversed again, etc... until signals for several positive and negative values of the longitudinal magnetization are obtained. Finally, either by hand or with the help of a microcomputer where the signals are stored for each measurement, the data are processed to extract the damping constant and the frequency of the decaying precession signals. The absolute value of this frequency, which depends primarily on the difference between the Larmor frequency of the nuclear spins and the reference frequency chosen for the lock-in amplifier, is not measured; what is actually measured is its linear dependence on $M$ which, according to theory, is given by $\mu M / \tau_{\mathrm{D}}$, as well as the diffusion time $\tau_{\mathrm{D}}$ which is directly obtained from the exponential decay of the signal. In practice, $\mu M / \tau_{\mathrm{D}}$ is of the order of $0.1 \mathrm{~Hz}$. The values of the relative polarization $M(-1 \leqslant M \leqslant 1)$ are obtained by auxiliary measurements with another set of pick-up coils connected in parallel, which allows extraction of the final value for $\mu$.

In order to have a better control of systematic errors, measurements were taken in various experimental conditions. Figure 2 shows the results for a series of cells, as compared to the theoretical results of reference [7] (full line). The triangles correspond to a cell filled with pure ${ }^{3} \mathrm{He}$; the squares to a cell with the same amount of ${ }^{3} \mathrm{He}$ (number density $n=10^{17} \mathrm{~cm}^{-3}$ ) plus $10 \%$ of ${ }^{4} \mathrm{He}$ added to the gas; the circles to again the same amount of ${ }^{3} \mathrm{He}$ plus $100 \%$ of ${ }^{4} \mathrm{He}$ added. Adding ${ }^{4} \mathrm{He}$ changes $\tau_{\mathrm{D}}$ and $\mu$ in the following way :

$$
\begin{aligned}
\tau_{\mathrm{D}} \rightarrow \tau_{\mathrm{D}}^{\prime} & =\tau_{\mathrm{D}}\left(D_{33}+D_{34}\right) / D_{33} \\
\mu \rightarrow \mu^{\prime} & =\mu D_{34} /\left(D_{33}+D_{34}\right)
\end{aligned}
$$

where $D_{33}$ is the spin diffusion coefficient on pure ${ }^{3} \mathrm{He}$ at the actual ${ }^{3} \mathrm{He}$ density (ignoring ${ }^{4} \mathrm{He}$ ) while $D_{34}$ the spin diffusion coefficient of a trace of ${ }^{3} \mathrm{He}$ in the actual density of ${ }^{4} \mathrm{He}$ (ignoring ${ }^{3} \mathrm{He}$ ).

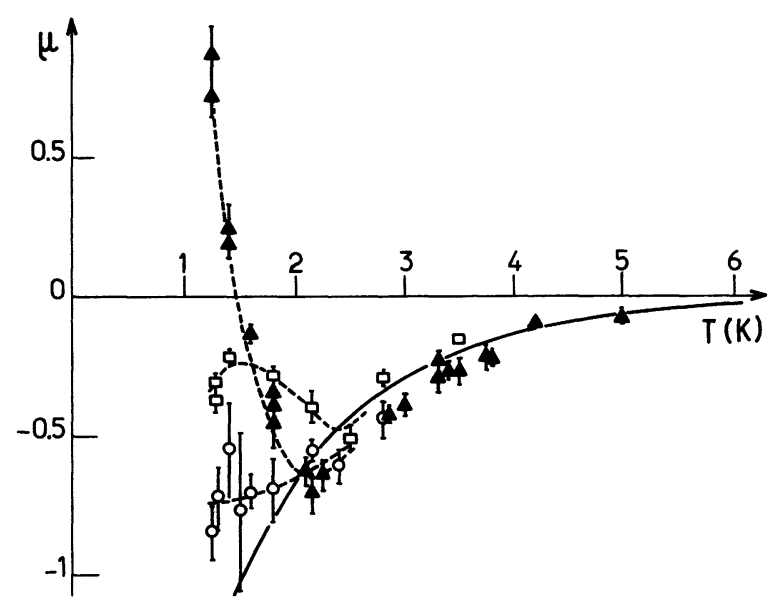

Fig. 2. - Measurements of $\mu$ obtained in a cell with pure ${ }^{3} \mathrm{He}(\Delta), 10 \%$ of ${ }^{4} \mathrm{He}$ added to ${ }^{3} \mathrm{He}(\square)$ or $100 \%$ added (O). The full line shows the theoretical calculation of reference [7]; the dashed lines are just a guide for the eye. Above $T=2.2 \mathrm{~K}$, the results are in good agreement between themselves and with theory; they confirm, with.more precision, the previous measurements reported in [11]. At lower temperatures, this agreement disappears, probably because of the formation of a ${ }^{3} \mathrm{He}$ monolayer on the surface of the solid $\mathrm{H}_{2}$ cryogenic coating. Only the measurements of $\mu$ above $2.2 \mathrm{~K}$ should be considered as valid. 
This is why, in figure 2 , the measured values $\mu^{\prime}$ have been multiplied by $\left(D_{33}+D_{34}\right) / D_{34}$, these diffusion coefficients being obtained from the calculations of reference [12]; in this way, all the points shown correspond to the same physical quantity $\mu$.

Clearly, the results at temperatures above $2.2 \mathrm{~K}$ are in good agreement with each other, as well as with theory. They are also in agreement with those of reference [11], obtained by an indirect detection method which might suffer from different sources of systematical errors : since the present detection method measures larger frequency shifts over shorter periods of time, possible $M$-dependent shifts introduced by parasitic effects are less important in relative value (for example, shifts introduced by spin diffusion in the tube connecting the measurement cell to the polarization cell, or by the magnetic image in the superconducting shield). The precision of the measurements was actually good and data were obtained more rapidly and more easily than with the indirect detection method; most of the error bars of figure 2 correspond actually to the uncertainty in the evaluation of $M$.

Between $1.2 \mathrm{~K}$, the lowest temperature accessible with our apparatus, and $2.2 \mathrm{~K}$, the measurements seem to lead to incompatible values of $\mu$. Possibly, $M$ dependent shifts due to magnetic dipole-dipole interactions could be introduced by the presence of an adsorbed phase of ${ }^{3} \mathrm{He}$ atoms on the surface of the solid hydrogen coating. According to the measurements of $\mathrm{V}$. LefèvreSeguin of the corresponding adsorption energy [13], $\Delta W \cong 13 \mathrm{~K}$; a monolayer should indeed be formed around $T \simeq \Delta W / 10$, in our experimental conditions, as discussed in [13], that is at about $T=1.5 \mathrm{~K}$. On the other hand, for ${ }^{3} \mathrm{He}-{ }^{4} \mathrm{He}$ mixtures, since ${ }^{4} \mathrm{He}$ atoms are preferentially adsorbed, they tend to expel the ${ }^{3} \mathrm{He}$ atoms from the walls, which could explain the strong effect of a relatively small amount of ${ }^{4} \mathrm{He}$ in the gas. On the other hand, we have not been able to develop a precise theory of these shifts (partly because they are observed over times of the order of $\tau_{\mathrm{D}}$, which are too short to use a motional narrowing theory). One can remark that similar difficulties also occur in the same temperature range with the indirect detection method : in that case, their manifestation is the appearance of strong slopes on diagrams such as the one shown in figure 2 of reference [11]. Consequently, only our measurements of $\mu$ above $T>2.2 \mathrm{~K}$ should be considered as real data.

In conclusion, the direct detection method gives more precise and presumably more reliable measurements of $\mu$ in ${ }^{3} \mathrm{He}$ between 2 and $5 \mathrm{~K}$. Since, in ${ }^{3} \mathrm{He}$, only relatively small values of $\mu M$ (not exceeding 0.5 ) are available with present techniques (while values ten times larger are obtained in $\mathrm{H} \uparrow$ [8]), measurements of $\mu$ are delicate and this independent measurement method is indeed welcome. Spin waves in degenerate liquid ${ }^{3} \mathrm{He}$ systems, where the Leggett theory [14] originated, have also been observed by various methods (Leggett-Rice effect on spin echoes [15] or other techniques making use of magnetic gradients [16, 17]). In one of them [18], a direct detection of a single diffusion mode was apparently obtained, but the reason for this mode selection is not completely clear, at least to the present authors. Finally, our results tend to show that NMR on the gas phase can be extremely sensitive to properties of an adsorbed phase. If this is confirmed and if the effects occurring at low temperatures can be better understood, this would open up the possibility of studying spin diffusion in adsorbed phases of ${ }^{3} \mathrm{He}$ on solid $\mathrm{H}_{2}$; comparison with theoretical calculations on spin diffusion in 2 dimensions $[19,20]$ would then become possible.

\section{References}

[1] " Spin polarized quantum systems ", J. Physique Colloq. 41 (1980) C-7 and references contained.

[2] Bashinin, E. P., Pis'ma Zh. Eksp. Teor. Fiz. 33 (1981) 8.

[3] Lhuillier, C. and Lalö̈, F., J. Physique 43 (1982) 197 and 225.

[4] Meyerovich, A. E., J. Low Temp. Phys. 53 (1983) 487. 
[5] Levy, L. P. and Ruckenstein, A. E., Phys. Rev. Lett. 52 (1984) 1512.

[6] LhUillier, C. and LALÖ̈, F., Comment on spin waves in dilute gases (unpublished).

[7] Lhuillier, C., J. Physique 44 (1983) 1.

[8] Johnson, B. R., Denker, J. S., Bigelow, N., Levy, L. P., Freed, J. H. and Lee, D. M., Phys. Rev. Lett. 52 (1984) 1508.

[9] Johnson, B. R., PhD Thesis, Cornell University (1984).

[10] Gully, W. J. and Mullin, W. J., Phys. Rev. Lett. 52 (1984) 1810.

[11] Nacher, P. J., Tastevin, G., Leduc, M., Crampton, S. B. and Lalö̈, F., J. Physique Lett. 45 (1984) L-441.

[12] LHUILLIER, C., private communication.

[13] LefÈVRe-Seguin, V., Thèse, Université Paris VI, (1984).

[14] Leggett, A. J., J. Phys. C 3 (1970) 448.

[15] Corrucini, L. R., Osheroff, D. D., Lee, D. D. and Richardson, R. C., Phys. Rev. Lett. 27 (1971) 650 ; J. Low Temp. Phys. 8 (1972) 229.

[16] Sholz, H. N., PhD Thesis, Ohio State University (1981).

[17] Masuhara, N., Candela, D., Edwards, D. O., Hoyt, R. F., Scholz, H. N., Sherrill, D. S. and Combescot, R., Phys. Rev. Lett. 53 (1984) 1168.

[18] Owers-Bradley, J. R., Chocholacs, H., Mueller, R. M., Buchal, Ch., Kubota, M. and Pobell, F., Phys. Rev. Lett. 51 (1983) 2120.

[19] MiYake, K. and Mullin, W. J., Phys. Rev. Lett. 50 (1983) 197 ; and J. Low Temp. Phys. 53 (1983) 313 ; MiYAKe, K., Phys. Rev. B 27 (1983) 3088.

[20] Lefèvre-Seguin, V., Guignes, H. et Lhuillier, C., to be submitted to J. Physique.

[21] Barbé, R., Leduc, M. et LaloË, F., J. Physique 35 (1974) 699. 